ISSN 0258-7122 (Print), 2408-8293 (Online)

Bangladesh J. Agril. Res. 44(1): 167-177, March 2019

\title{
EVALUATION OF JACKFRUIT GERMPLASM FOR YIELD AND YIELD CONTRIBUTING CHARACTERS
}

\author{
M. Z. RAHMAN ${ }^{1}$, M. R. KARIM ${ }^{2}$, M. A. HAQUE ${ }^{3}$ AND M. J. RAHMAN ${ }^{4}$
}

\begin{abstract}
Twenty germplasm of jackfruit grown in the Fruit Research Farm of Horticulture Research Centre (HRC), Bangladesh Agricultural Research Institute (BARI) were evaluated for growth, yield and yield parameter along with qualitative characters from July 2012 to June 2015. All the germplasm varied for most of the parameters evaluated. The tallest plant was recorded in AH Joy-079 $(6.75 \mathrm{~m})$ and shortest plant in AH Joy-075 $(3.50 \mathrm{~m})$ among the germplasm. Base girth ranged from $42.50 \mathrm{~cm}$ to $80.20 \mathrm{~cm}$ in AH Joy-075 and AH Joy-085, respectively. Maximum plant spreading with North-South and East-West canopy (5.50 m and $6.00 \mathrm{~m}$, respectively) was recorded in AH Joy079 and minimum $(3.00 \mathrm{~m}$ and $3.50 \mathrm{~m})$ in both AH Joy-075 and AH Joy-090 germplasm. The fruit number varied from 02 to 11 among the germplasm. Individual fruit weight ranged from $2.27 \mathrm{~kg}$ to $11.33 \mathrm{~kg}$ where AH Joy-034 had the lowest and AH Joy-092 noted the highest fruit weight. Maximum bulb (184) was recorded in AH Joy-092 and minimum (45) in AH Joy-085. Maximum TSS (25.0) was recorded in AH Joy-029 and minimum (14.0) in AH Joy-067 and the edible portion was noted the highest in AH Joy-036 (48.60\%) where it was lowest in AH Joy-078 (32.10\%). Bulb texture was soft to hard. Maximum germplasm were juicy to medium juicy having yellow to light yellowPulp. Therefore, The germplasm AH Joy-089, AH Joy-017 and AH Joy-092 may be selected for higher yielder. The germplasm AH Joy-034,AH Joy-067, AH Joy078,AH Joy-085 and AH Joy-098 should be selected for family size jackfruit as their individual fruit weight ranges between 2 to $4 \mathrm{~kg}$ including yellow bulb colourcontaining good juiciness and soft bulb texture.
\end{abstract}

Keywords: Jackfruit, qualitative characters, quantitative characters, family size fruit and yield.

\section{Introduction}

Jackfruit (Artocarpus heterophyllus L.) is a tree species of the family Moraceae which is native to Southeast Asia. It is the largest edible fruit in the world (Naik, 1949 and Sturrock, 1959). Jackfruit locally called "kathal" has gained the position of national fruit of Bangladesh due to its popularity and diversified use and other features. Every part of either the jackfruit tree or fruit has great economic importance. The poor people of jackfruit growing area used to eat this fruit instead of rice for one of their daily meals, in this regard it is commonly referred to as "the poor man's food" (Rahman et al., 1994). It is a cross-

${ }^{1 \& 4}$ Senior Scientific Officer, Horticulture Research Centre (HRC), Bangladesh Agricultural Research Institute (BARI), Gazipur-1701, ${ }^{2 \& 3}$ Scientific Officer, HRC, BARI, Gazipur-1701, Bangladesh. 
pollinated fruit tree and is mainly propagated by seeds in Bangladesh. As a result, wide range of variation is exhibited in morpho-agronomic characteristics. Narasimham (1990) and Hossain (1996) reported different types of jackfruit, which vary widely in size, shape, bearing habit and quality. Azad (1999) noted the vast genetic diversity in jackfruit on the basis of morpho-agronomic traits and using isozyme marker.Jackfruit is identified as deserving priority attention in Bangladesh because of its importance and diversity (Azad et al., 2007; Hossain, 1996; Saha et al., 1996), and Bangladesh is considered as a secondary center of jackfruit diversity (Arora, 1998; Dhar, 1998 and Hossain, 1996). Jackfruit rips mostly in May-July and there is an abundance of fruit in the market in this period. Every year a lot of fruits get rotten at that time. If the farmers can produce jackfruit early, they can get more benefit. It is a large monoecious tree producing fruit up to $50 \times 100 \mathrm{~cm}$ size and weighing $50 \mathrm{~kg}$ (Jagadeesh et al., 2006). The development of jackfruit varieties through hybridization is cumbersome and more time consuming. Therefore, selection of superior chance seedling has been suggested for the varietal development of jackfruit. The field population of jackfruit is composed of a large number of variable genotypes providing ample scope of selecting the superior ones.Bangladesh Agricultural Research Institute has already released two jackfruit varieties, but still there are opportunities to develop jackfruit varieties that farmers can cultivate for better yield and quality. Keeping this view in mind, the study was therefore, carried out to find out suitable line(s) for the development of a variety.

\section{Materials and Methods}

The studywas conducted at the Fruit Research Farm of Horticulture Research Centre (HRC), Bangladesh Agricultural Research Institute (BARI) during June

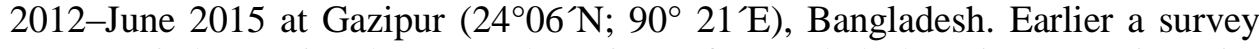
was carried out in the central region of Bangladesh using questionnaire developed as per descriptor of jackfruit published by International Plant Genetic Resources Institute (IPGRI, 2000). Fruits of 20 germplasm were collected and five fruits from each accession were analyzed in the laboratory to study the fruit characteristics. Seedlings of each accession were planted following single row plantation in the Fruit Research Farm of HRC on 01 July 2001. The age of plants was 10 years at the time of study. Subsequently three fruiting years of each accession are included under this study. The climate of the experimental area was under subtropical zone which was characterized by high temperature and high rain during April to November and little or no rain during October to march. The soil of the experimental site was clay loam belonging to the Modhupur tract under Agro Ecological Zone 28 (Ahmed et al., 2016). Planting distance was maintained $6 \mathrm{~m} \times 3 \mathrm{~m}$. A fertilizer dose of 300g Urea, 300g TSP, 250g MOP and $20 \mathrm{~kg}$ cowdung per plant and subsequent irrigation were applied on August 2012 after harvesting of fruits. The experimental plot was ploughed with power tiller just before applying fertilizer. Weeding was performed in the month March and April 2013. Unwanted branches were prunned off in September 2012. Data on 
plant height, base girth, canopy spreading (north-south and east-west spread), number of fruits per plant, fruit length and diameter, spine number per 5 square $\mathrm{cm}$, rind weight, rachis weight, bulb length and width, TSS, number of bulb and edible portion were recorded and analyzed statistically. Tree volume was calculated using the formula of Westwood et al. (1963), i.e., tree volume $\left(\mathrm{m}^{3}\right)_{=} 4 / 3 \pi \mathrm{a}^{2} \mathrm{~b}$, where $\mathrm{a}=$ average of east-west and north-south spread, $b=$ half of plant height and $\pi$ (constant) $=3.14$.

\section{Results and Discussion}

\section{Growth characteristics of jackfruit germplasm}

Plant and fruit characteristics of 20 accessions of jackfruit are presented in Table 1. The highest plant height $(6.75 \mathrm{~m})$ was found in AH Joy-079 closely followed by AH Joy-086 (6.50 m) and AH Joy-059 $(6.25 \mathrm{~m})$, while it was noted the lowest (3.50 m) in AH Joy-075.

Table 1. Growth characteristics of 20 jackfruit germplasm (average of 3 years)

\begin{tabular}{c|c|c|c|c|c}
\hline \multirow{2}{*}{ Acc. no. } & $\begin{array}{c}\text { Plant height } \\
(\mathrm{m})\end{array}$ & $\begin{array}{c}\text { Base girth } \\
(\mathrm{cm})\end{array}$ & \multicolumn{2}{|c|}{ Canopy Spreading } & Tree volume \\
\cline { 4 - 5 } & & $\mathrm{N}-\mathrm{S}$ & $\mathrm{E}-\mathrm{W}$ & $\left(\mathrm{m}^{3}\right)$ \\
\hline AH Joy-017 & 5.50 & 75.00 & 4.50 & 4.75 & 16.96 \\
AH Joy-029 & 6.00 & 60.00 & 4.00 & 5.25 & 18.50 \\
AH Joy-032 & 6.00 & 62.50 & 3.75 & 4.00 & 15.50 \\
AH Joy-034 & 5.67 & 60.00 & 3.00 & 4.33 & 13.85 \\
AH Joy-036 & 5.55 & 55.60 & 3.10 & 4.00 & 13.14 \\
AH Joy-059 & 6.25 & 72.00 & 4.75 & 5.00 & 20.31 \\
AH Joy-067 & 5.50 & 66.00 & 3.50 & 4.25 & 14.21 \\
AH Joy-075 & 3.50 & 42.50 & 3.00 & 3.50 & 18.90 \\
AH Joy-078 & 5.30 & 65.00 & 5.70 & 5.00 & 7.58 \\
AH Joy-079 & 6.75 & 55.80 & 5.50 & 6.00 & 25.88 \\
AH Joy-080 & 5.67 & 45.50 & 4.70 & 4.00 & 16.44 \\
AH Joy-081 & 4.75 & 68.30 & 5.00 & 4.85 & 15.60 \\
AH Joy-085 & 5.33 & 80.20 & 4.65 & 5.20 & 17.50 \\
AH Joy-086 & 6.50 & 50.50 & 4.50 & 5.33 & 21.30 \\
AH Joy-087 & 3.75 & 58.00 & 3.75 & 4.25 & 10.00 \\
AH Joy-089 & 5.75 & 75.50 & 5.50 & 5.80 & 21.66 \\
AH Joy-090 & 4.30 & 45.00 & 3.00 & 3.50 & 9.32 \\
AH Joy-092 & 4.50 & 55.00 & 4.00 & 3.75 & 11.63 \\
AH Joy-098 & 5.20 & 65.00 & 3.50 & 4.30 & 13.52 \\
AH Joy-097 & 4.33 & 58.00 & 4.00 & 4.55 & 12.34 \\
\hline Mean & 5.30 & 60.77 & 4.18 & 4.58 & 15.71 \\
SD & 0.87 & 10.43 & 0.85 & 0.72 & 4.23 \\
CV (\%) & 16.51 & 17.16 & 20.30 & 15.63 & 12.43 \\
\hline
\end{tabular}


Maximum base girth $(80.20 \mathrm{~cm})$ was obtained from AH Joy-085 followed by AH Joy-089 $(75.50 \mathrm{~cm})$ and AH Joy-017 $(75.00 \mathrm{~cm})$. Minimum base girth $(42.50 \mathrm{~cm})$ was found in AH Joy-075. North-South $(5.50 \mathrm{~m})$ and east-west $(6.00 \mathrm{~m})$ plant spreading were exhibited maximum in AH Joy-079 and those were demonstrated minimum (3.00 m and $3.50 \mathrm{~m}$, respectively) in AH Joy-090. More or less similar physico-morphological variation has been reported in jackfruit by Reddy et al., 2004; Shyamalamma et al., 2008; Ullah and Haque, 2008. Maximum tree volume was yielded by the germplasm AH Joy-079 $\left(25.88 \mathrm{~m}^{3}\right)$ where it was minimum $\left(7.58 \mathrm{~m}^{3}\right)$ in AH Joy-078.

\section{Fruit characteristics of jackfruit germplasm}

\section{a. Quantitative characteristics}

A wide variation in physico-morphological fruit characteristics of jackfruit was observed among the 20 jackfruit germplasm (Table 2). The harvesting time showed that first harvesting was done in all the germplasm from mid-May to late May in three consecutive study years. The earliest first harvest (12-16 May) was recorded in AH Joy-098 which was late (26-31 May) in the germplasm AH Joy-017.Duration of harvest was carried out 24.67 to 48.67 days (in the germplasm AH Joy-087 and AH Joy-080 respectively) which is supported by the result of karim et al. (2008). Maximum coefficient of variation was observed for number of bulbs/fruit(50.41\%) followed by number of fruits per plant $(44.81 \%)$.Bulbis the economically important part of jackfruit as it comprises the edible part known as flake. Hence these characters can be considered for selection of elite type jackfruit. There are a few published reports which indicated variation for bulb characters in jackfruit clones (Muralidharan et al., 1997; Mitra and Mani, 2000; Reddy et al., 2004 and Jagadeesh et al., 2010). Total soluble solids in the genotypes recorded comparatively low values for coefficient of variation (14.75\%) signifying that this trait is conserved in nature. This result agreed with findings of Jagadeesh $e t$ al. (2010). However, a slight variation in this parameter can cause considerable impact on taste. Maximum number of fruits per plant (10.67) was produced by the accession AH Joy-067 followed by AH Joy-017 (8.33) and AH Joy-089 (7.67) whereas minimum number (02) was found in AH Joy-081 and AH Joy087.The biggest fruit $(11.33 \mathrm{~kg})$ with maximum fruit length $(39.00 \mathrm{~cm})$ and diameter $(25.20 \mathrm{~cm})$ was harvested from the germplasm AH Joy-092 followed by AH Joy-090 $(8.63 \mathrm{~kg})$.

The smallest fruit $(2.27 \mathrm{~kg})$ having minimum fruit length $(23.50 \mathrm{~cm})$ and diameter $(13.80 \mathrm{~cm})$ was observed in AH Joy-034. Very dense spine (per five squire centimeter) was found in AH Joy-090 (151) followed by AH Joy-089 (134) and minimum in AH Joy-017 (38). 
Table 2. Fruit characteristics of 20 jackfruit germplasm (average of 3 years)

\begin{tabular}{c|c|c|c|c|c|c|c|c}
\hline Acc. no. & $\begin{array}{c}\text { First } \\
\text { harvesting } \\
\text { time }\end{array}$ & $\begin{array}{c}\text { Duration } \\
\text { of harvest } \\
(\text { days })\end{array}$ & $\begin{array}{c}\text { Fruit } \\
\text { length } \\
(\mathrm{cm})\end{array}$ & $\begin{array}{c}\text { Fruit dia } \\
(\mathrm{cm})\end{array}$ & $\begin{array}{c}\text { Spine } \\
\text { no/5sq. } \\
\text { cm of } \\
\text { fruit }\end{array}$ & $\begin{array}{c}\text { No of } \\
\text { bulbs/f } \\
\text { ruit }\end{array}$ & $\begin{array}{c}\text { TSS } \\
(\%)\end{array}$ & $\begin{array}{c}\text { Edible } \\
\text { portion } \\
(\%)\end{array}$ \\
\hline AH Joy-017 & 26-31 May & 41.00 & 25.0 & 24.4 & 38 & 75 & 20.0 & 42.50 \\
AH Joy-029 & 20-25 May & 39.33 & 30.4 & 23.0 & 63 & 181 & 25.0 & 43.20 \\
AH Joy-032 & 22-27 May & 40.33 & 25.0 & 20.2 & 59 & 60 & 20.0 & 39.20 \\
AH Joy-034 & 16-20 May & 28.67 & 23.5 & 13.8 & 103 & 94 & 21.0 & 44.70 \\
AH Joy-036 & 15-20 May & 36.33 & 28.1 & 23.0 & 64 & 104 & 16.0 & 48.60 \\
AH Joy-059 & 15-20 May & 42.00 & 26.1 & 21.8 & 64 & 73 & 15.5 & 48.20 \\
AH Joy-067 & 18-24 May & 37.67 & 26.3 & 16.2 & 87 & 74 & 14.0 & 41.40 \\
AH Joy-075 & 15-22 May & 46.33 & 26.1 & 24.1 & 47 & 165 & 18.0 & 44.60 \\
AH Joy-078 & 18-22 May & 34.67 & 29.7 & 17.0 & 64 & 52 & 14.0 & 32.10 \\
AH Joy-079 & 15-20 May & 46.33 & 33.2 & 20.1 & 108 & 55 & 16.5 & 45.50 \\
AH Joy-080 & 16-22 May & 48.67 & 26.5 & 21.8 & 100 & 182 & 18.0 & 40.00 \\
AH Joy-081 & 20-25 May & 30.67 & 30.3 & 24.0 & 102 & 130 & 16.0 & 41.80 \\
AH Joy-085 & 24-30 May & 46.00 & 34.3 & 19.3 & 69 & 45 & 17.0 & 37.20 \\
AH Joy-086 & 20-27 May & 40.67 & 33.7 & 18.7 & 49 & 175 & 20.0 & 39.70 \\
AH Joy-087 & 22-28 May & 24.67 & 35.2 & 18.2 & 60 & 45 & 16.0 & 32.00 \\
AH Joy-089 & 15-20 May & 45.67 & 32.0 & 20.7 & 134 & 56 & 16.5 & 44.20 \\
AH Joy-090 & 15-22 May & 49.33 & 37.5 & 23.0 & 151 & 157 & 18.0 & 43.90 \\
AH Joy-092 & 20-27 May & 26.33 & 39.0 & 25.2 & 57 & 184 & 20.0 & 45.10 \\
AH Joy-098 & 12-16 May & 36.33 & 23.7 & 17.0 & 66 & 63 & 18.5 & 47.90 \\
AH Joy-097 & 18-25 May & 42.67 & 26.6 & 22.5 & 48 & 165 & 20.0 & 42.60 \\
\hline Mean & - & 39.18 & 29.36 & 20.70 & 76.65 & 106.75 & 18.00 & 42.22 \\
SD & - & 7.29 & 4.24 & 3.13 & 31.51 & 53.81 & 2.66 & 4.60 \\
TSS & - & 18.59 & 14.44 & 15.11 & 41.10 & 50.41 & 14.75 & 10.90 \\
\hline
\end{tabular}

TSS $=$ Total Soluble Solid

Maximum TSS (25.0) was recorded in AH Joy-029 and minimum (14.0) in AH Joy-067 with a mean value of 18.0 which is agreed with the findings of Rahman et al. (1994). The edible portion was recorded the highest in AH Joy-036 (48.6\%) followed by AH Joy-059 (48.2\%) and the lowest in AH Joy-078 (32.1\%). The findings of the study partially similar to the results of Ullah and Haque (2008) where the authors reported 37.3 to $52.8 \%$ edible portion in their studied germplasm. Maximum bulb was counted in AH Joy-092 (184) followed by AH Joy-080 (182) and minimum in AH Joy-085 (45). 
Table 2. Continued

\begin{tabular}{|c|c|c|c|c|c|c|c|c|c|c|}
\hline \multirow[b]{2}{*}{ Acc. no. } & \multicolumn{4}{|c|}{ Individual fruit wt (kg) } & \multicolumn{4}{|c|}{ Fruits/ plant (no.) } & \multirow{2}{*}{$\begin{array}{c}\text { Rind } \\
\text { wt (kg) }\end{array}$} & \multirow{2}{*}{$\begin{array}{l}\text { Rachis } \\
\text { wt (g) }\end{array}$} \\
\hline & 2012-13 & 2013-14 & 2014-15 & Average & $\begin{array}{c}2012- \\
13\end{array}$ & $\begin{array}{c}2013- \\
14\end{array}$ & $\begin{array}{c}2014- \\
15\end{array}$ & Average & & \\
\hline AH Joy-017 & 4.60 & 5.50 & 5.90 & 5.33 & 7 & 10 & 8 & 8.33 & 3.40 & 225 \\
\hline AH Joy-029 & 6.65 & 7.40 & 8.60 & 7.55 & 4 & 4 & 5 & 4.33 & 4.00 & 260 \\
\hline AH Joy-032 & 4.40 & 5.80 & 5.00 & 5.07 & 6 & 5 & 7 & 6.00 & 2.80 & 240 \\
\hline AH Joy-034 & 2.20 & 2.20 & 2.40 & 2.27 & 4 & 6 & 5 & 5.00 & 1.10 & 150 \\
\hline AH Joy-036 & 7.00 & 7.10 & 7.00 & 7.03 & 3 & 4 & 5 & 4.00 & 3.10 & 500 \\
\hline AH Joy-059 & 5.30 & 5.00 & 6.25 & 5.52 & 7 & 6 & 6 & 6.33 & 2.60 & 200 \\
\hline AH Joy-067 & 3.00 & 2.60 & 3.20 & 2.93 & 11 & 11 & 10 & 10.67 & 1.40 & 300 \\
\hline AH Joy-075 & 6.45 & 6.50 & 6.70 & 6.55 & 3 & 4 & 3 & 3.33 & 3.30 & 300 \\
\hline AH Joy-078 & 3.00 & 3.30 & 3.10 & 3.13 & 6 & 7 & 7 & 6.67 & 1.90 & 185 \\
\hline AH Joy-079 & 6.40 & 7.30 & 8.00 & 7.23 & 3 & 2 & 4 & 3.00 & 3.80 & 165 \\
\hline AH Joy-080 & 6.00 & 6.50 & 6.50 & 6.33 & 3 & 5 & 4 & 4.00 & 3.50 & 280 \\
\hline AH Joy-081 & 6.00 & 6.30 & 6.20 & 6.17 & 2 & 2 & 3 & 2.33 & 3.20 & 350 \\
\hline AH Joy-085 & 4.00 & 4.50 & 4.20 & 4.23 & 7 & 7 & 8 & 7.33 & 2.50 & 170 \\
\hline AH Joy-086 & 4.90 & 5.40 & 5.20 & 5.17 & 4 & 6 & 6 & 5.33 & 2.80 & 250 \\
\hline AH Joy-087 & 4.50 & 5.00 & 5.00 & 4.83 & 3 & 2 & 2 & 2.33 & 3.00 & 300 \\
\hline AH Joy-089 & 5.40 & 6.10 & 6.00 & 5.83 & 8 & 8 & 7 & 7.67 & 3.00 & 275 \\
\hline AH Joy-090 & 8.50 & 8.80 & 8.60 & 8.63 & 4 & 3 & 5 & 4.00 & 4.20 & 550 \\
\hline AH Joy-092 & 11.00 & 11.50 & 11.50 & 11.33 & 3 & 3 & 4 & 3.33 & 5.80 & 500 \\
\hline AH Joy-098 & 3.50 & 3.85 & 3.55 & 3.63 & 6 & 5 & 7 & 6.00 & 1.60 & 250 \\
\hline AH Joy-097 & 6.50 & 7.00 & 7.00 & 6.83 & 4 & 6 & 5 & 5.00 & 3.70 & 200 \\
\hline Mean & 5.465 & 5.882 & 5.995 & 5.78 & 4.9 & 5.3 & 5.55 & 5.25 & 3.04 & 282.50 \\
\hline SD & 2.040 & 2.141 & 2.197 & 2.108 & 2.27 & 2.49 & 1.99 & 2.16 & 1.06 & 113.92 \\
\hline CV (\%) & 37.33 & 36.39 & 36.64 & 36.47 & 46.30 & 47.06 & 35.79 & 41.09 & 34.96 & 40.33 \\
\hline
\end{tabular}

* Three years average data of rind weight and rachis weightare shown in the table.

The highest rind weight $(5.8 \mathrm{~kg}$ ) was recorded in $\mathrm{AH}$ Joy-092 followed by $\mathrm{AH}$ Joy-090 (4.2 kg). The lowest rind weight was noticed in AH Joy-034 (1.1 kg). Maximum rachis weight (550 g) was observed in AH Joy-090 followed by AH Joy-036 and AH Joy-092 (500 g) while it was recorded minimum in AH Joy-034 $(150 \mathrm{~g})$. 
Table 3. Yield performance of 20 jackfruit germplasm

\begin{tabular}{|c|c|c|c|c|}
\hline \multirow{2}{*}{ Acc. no. } & \multicolumn{4}{|c|}{ Yield/ plant (kg) } \\
\hline & 2012-13 & 2013-14 & 2014-15 & Average \\
\hline AH Joy-017 & 32.20 & 55.00 & 47.20 & 44.44 \\
\hline AH Joy-029 & 26.60 & 29.60 & 43.00 & 32.72 \\
\hline AH Joy-032 & 26.40 & 29.00 & 35.00 & 30.40 \\
\hline AH Joy-034 & 8.80 & 13.20 & 12.00 & 11.33 \\
\hline AH Joy-036 & 21.00 & 28.40 & 35.00 & 28.13 \\
\hline AH Joy-059 & 37.10 & 30.00 & 37.50 & 34.94 \\
\hline AH Joy-067 & 33.00 & 28.60 & 32.00 & 31.29 \\
\hline AH Joy-075 & 19.35 & 26.00 & 20.10 & 21.83 \\
\hline AH Joy-078 & 18.00 & 23.10 & 21.70 & 20.89 \\
\hline AH Joy-079 & 19.20 & 14.60 & 32.00 & 21.70 \\
\hline AH Joy-080 & 18.00 & 32.50 & 26.00 & 25.33 \\
\hline AH Joy-081 & 12.00 & 12.60 & 18.60 & 14.39 \\
\hline AH Joy-085 & 28.00 & 31.50 & 33.60 & 31.04 \\
\hline AH Joy-086 & 19.60 & 32.40 & 31.20 & 27.56 \\
\hline AH Joy-087 & 13.50 & 10.00 & 10.00 & 11.28 \\
\hline AH Joy-089 & 43.20 & 48.80 & 42.00 & 44.72 \\
\hline AH Joy-090 & 34.00 & 26.40 & 43.00 & 34.53 \\
\hline AH Joy-092 & 33.00 & 34.50 & 46.00 & 37.78 \\
\hline AH Joy-098 & 21.00 & 19.25 & 24.85 & 21.80 \\
\hline AH Joy-097 & 26.00 & 42.00 & 35.00 & 34.17 \\
\hline Mean & 24.50 & 28.37 & 31.29 & 28.01 \\
\hline SD & 8.99 & 11.53 & 10.80 & 9.57 \\
\hline $\mathrm{CV}(\%)$ & 36.71 & 40.62 & 34.53 & 34.15 \\
\hline
\end{tabular}

Number of fruit (10.67) was highest in AH Joy-067 followed by AH Joy-017 (8.33) and AH Joy-089 (7.67) but the highest yield per plant was calculated in AH Joy-089 (44.72 kg/plant) followed by AH Joy-017 (44.45 kg/plant) and the lowest yield per plant was in AH Joy-034 and Ah Joy-087 (11.28 kg/plant).

\section{b. Qualitative characteristics}

Out of twenty germplasm, maximum (9) gave ellipsoid shaped fruit and others were clavate, spheroid and oblong in shaped(Table-4). Fruit colour varied from 
yellow to greenish yellow. Fruit surface of maximum germplasm was spiny except three (AH Joy-032, AH Joy-089, AH Joy-097) which showed smooth fruit surface. Spine shape was Intermediate to pointed. Regarding attractiveness of fruit four germplasm (AH Joy-029, AH Joy-032, AH Joy-059 and AH Joy-097) produced good fruits and others produced intermediate to poor fruits. Pulps were yellow to light yellow in colour. Bulb of maximum germplasm were juicy to medium juicy except AH Joy-089 and AH Joy-092 which were less juicy. Bulb texture was soft to hard and only two germplasm (AH Joy-017 and AH Joy-086) showed high fibrousness and others were medium and low. Most of the studied characters like fruit colour, fruit shape and pulp colour are similar to the research results of Azad et al. (2001) and Roy et al. (2012).

Table 4. Qualitative characteristics of jackfruit germplasm

\begin{tabular}{|c|c|c|c|c|c|}
\hline Acc. no. & Fruit Shape & Fruit colour & Fruit surface & Spine shape & Attractiveness \\
\hline AH Joy-017 & Clavate & Greenish yellow & Spiny & Intermediate & Intermediate \\
\hline AH Joy-029 & Ellipsoid & Yellow & Spiny & Intermediate & Good \\
\hline AH Joy-032 & Oblong & Yellow & Smooth & Intermediate & Good \\
\hline AH Joy-034 & Oblong & Greenish yellow & Spiny & Intermediate & Poor \\
\hline AH Joy-036 & Spheroid & Yellow & Spiny & Intermediate & Intermediate \\
\hline AH Joy-059 & Spheroid & Greenish yellow & Spiny & Intermediate & Good \\
\hline AH Joy-067 & Ellipsoid & Yellow & Spiny & Pointed & Poor \\
\hline AH Joy-075 & Spheroid & Yellow & Spiny & Intermediate & Intermediate \\
\hline AH Joy-078 & Ellipsoid & Yellow & Spiny & Pointed & Intermediate \\
\hline AH Joy-079 & Oblong & Yellow & Spiny & Intermediate & Intermediate \\
\hline AH Joy-080 & Ellipsoid & Yellow & Spiny & Intermediate & Intermediate \\
\hline AH Joy-081 & Clavate & Greenish yellow & Spiny & Pointed & Intermediate \\
\hline AH Joy-085 & Ellipsoid & Yellow & Spiny & Pointed & Intermediate \\
\hline AH Joy-086 & Oblong & Yellow & Spiny & Intermediate & Intermediate \\
\hline AH Joy-087 & Oblong & Greenish yellow & Spiny & Intermediate & Poor \\
\hline AH Joy-089 & Ellipsoid & Yellow & Smooth & Intermediate & Intermediate \\
\hline AH Joy-090 & Ellipsoid & Yellow & Spiny & Intermediate & Intermediate \\
\hline AH Joy-092 & Ellipsoid & Greenish yellow & Spiny & Intermediate & Intermediate \\
\hline AH Joy-098 & Ellipsoid & Yellow & Spiny & Intermediate & Intermediate \\
\hline AH Joy-097 & Spheroid & Greenish yellow & Smooth & Intermediate & Good \\
\hline
\end{tabular}


Table 4. Continued

\begin{tabular}{rc|c|c|c}
\hline Acc. no. & Bulb color & Bulb Juiciness & Bulb fibrousness & Bulb texture \\
\hline AH Joy-017 & Yellow & Juicy & High & Hard \\
AH Joy-029 & Yellow & Juicy & Low & Soft \\
AH Joy-032 & Yellow & Juicy & Low & Soft \\
AH Joy-034 & Yellow & M juicy & Medium & Soft \\
AH Joy-036 & Yellow & Juicy & Low & Soft \\
AH Joy-059 & Yellow & M juicy & Low & Soft \\
AH Joy-067 & yellow & Juicy & Low & Soft \\
AH Joy-075 & yellow & Juicy & Medium & Hard \\
AH Joy-078 & L yellow & Juicy & Low & Semi hard \\
AH Joy-079 & Yellow & Juicy & Medium & Hard \\
AH Joy-080 & Yellow & Juicy & Low & Soft \\
AH Joy-081 & L yellow & Juicy & Low & Soft \\
AH Joy-085 & Yellow & Juicy & Low & Soft \\
AH Joy-086 & Yellow & Juicy & High & Soft \\
AH Joy-087 & L yellow & Juicy & Low & Soft \\
AH Joy-089 & L yellow & L juicy & Medium & Hard \\
AH Joy-090 & Yellow & Juicy & Low & Semi hard \\
AH Joy-092 & Yellow & L juicy & Low & Soft \\
AH Joy-098 & L yellow & M juicy & Low & Semi hard \\
AH Joy-097 & Yellow & M juicy & Medium & Soft \\
\hline
\end{tabular}

*L Yellow=Light yellow; L Juicy=Less Juicy; M Juicy=Medium Juicy

\section{Conclusion}

Evaluation of available jackfruit germplasm helps to developnew varieties. Keeping this view in mind, the evaluation can be concluded with the selection of AH Joy-089, AH Joy-017 and AH Joy-092 for top yielder but AH Joy-034,AH Joy-067, AH Joy-078,AH Joy-085 and AH Joy-098germplasmshould be selected for the family size fruits.

\section{References}

Ahmed, R., M. R. Karim, S. Ahmed, M. A. Quddus and M. A. Siddiky. 2016. Response of gladiolus to combined application of nitrogen, phosphorus, potassium and sulfur in grey terrace soil of gazipur, Bangladesh. Ecofriendly Agril. J. 9(12) Pp.93-97.

Arora, R. K. 1998. Genetic Resources of Native Tropical Fruits in Asia: Diversity, Distribution and IPGRI's Emphasis on the Conservation and Use. Pp 42-53 In R. K. Arora and V. R. Rao, eds., Tropical Fruits in Asia-Diversity, Maintenance, Conservation and Use. International Plant Genetic Resource Institute, New Delhi.

Avishek Roy, Pratibha, Manoj Chaudhry and Ankit Dongariyal. 2018. Studies on Morphological Traits of Jackfruit (Artocarpusheterophyllus Lam.) Germplasm under 
tarai Conditions of Uttarakhand, India. Int. J. Curr. Microbiol. App. Sci. 7(01):31193125.doi: https://doi.org/10.20546/ijcmas.2018.701.370

Azad A. K, J. G Jones and N.Haq 2007.Assessing morphological and isozyme variation of jackfruit (Artocarpus heterophyllus Lam.) in Bangladesh. Agroforestry Syst. 71: 109-125.

Azad, A. K. 1999. Genetic diversity of jackfruit in Bangladesh and development of propagation methods. $\mathrm{Ph}$ D thesis, University of Southampton.UK, 200p.

Azad, A. K., Majumder, A. A. Saha, M. G and Ahmad M. R. 2001.Research Report on Fruit Improvement, Pomology Division, HRC, BARI, Gazipur, Pp-1-14.

Dhar, M. 1998. Techniques of Vegetative and in vitro Propagation of Jackfruit.Institute of Postgraduate Studies in Agriculture, Salna, Gazipur, Bangladesh.

Gomez, K. A. and Gomez, A.A 1984.Statistical Procedures of Agricultural Research. $2^{\text {nd }}$ Edn. John Willey and Sons, New York. Pp. 97-411.

Hossain, A. K. M. A. 1996. Status reports on genetic resource of Jackfruit in Bangladesh. IPGRI Regional office, Singapore, P.30.

IPGRI, 2000. Descriptor for jackfruit (Artocarpus heterophyleus). IPGRI office for South Asia.National Bureau of Plant Genetic Resources, PUSA campus, New Delhi 110 012. India. P-64.

Jagadeesh, S. L., B. S. Reddy, G. S. K. Swamy, K. Gorbal, L.Hegde, and G. S. V. Raghaven. 2006. Chemical Composition of Jackfruit (Artocarpus heterophyllus Lam.) Selections of Western Ghats of India. Food Chemistry 102:361-365.

Jagadeesh, S.L., B.S.Reddy, N.Basavaraj, G.S.K. Swamy, and L. Hegde,. 2010. Variability studies in physic-chemical qualities of jack fruit (Artocarpus heterophyllus Lam.) of coastal zone of Karnataka. Karnataka J. Agric. Sci., 23: 293-297.

Karim M.R., Haque M.A., Yasmin L., Nazim Uddin M. and Haque A.H.M.M. 2008.Effect of Harvesting Time and Varieties on the Physicochemical Characteristics of Jackfruits (Artocarpus heterophyllus Lam.). Int. J. Sustain. Crop Prod. 3(6):48-57.

Mitra, S.K. and D.Mani, 2000.Conversation and utilization of genetic resources in jackfruit (Artocarpus heterophyllus Lam.) - A potential underutilized fruit. Acta Hort., 523: 229-232.

Muralidharan, V.K., M. M. Ganapathy, K.C. Velayudhan, and A. Rai 1997. Collecting jackfruit germplasm in Western Ghats. Indian J. Pl. Gen. Res., 10: 227-231.

Narasimham, P. 1990. Breadfruit and jackfruit.In: S. Nagy, P. E. Shaw and W. F. Wardowski (eds). Fruits of Tropical and Subtropical Origin-Composition, properties and uses, Florida: Florida Science Source, Inc. Pp.216-259.

Naik. K. C. 1949. South Indian Fruits and Their Culture. P. Varadachery \& Co. Madras. Pp. 300-302.

Rahman, M.J., M.A.Haque, and M.S. Haque, 1994. Physicochemical characteristics of different type's jackfruit during storage.Bangladesh Horticulture. 22(1\&2): 75-83.

Reddy, B.M.C., P.Patil, S.Shashikumar, and L.R.Govindaraju, 2004. Studies on physicochemical characters of jack fruit clones of South Karnataka. Karnataka J. Agric. Sci., 17: 279-282. 
Roy, C. A., A. K. Bhattacharyya and P. Chattopadhyay. 2012. Study on functional properties of raw and blended jackfruit seed flour (a non-conventional source) for food application. Indian Journal of Natural Products and Resources. Vol. 3(3), September 2012, Pp. 347-353.

Saha, M. C., M. G. Sha, M. A. Rahman, M. I. Nazrul, A. Quasem, N. K. Halder, and A. F. M. E. Haque. 1996. Variability in Jackfruit, Internal Research Review Workshop, Horticulture Research Center, BARI, Gazipur. Pp.1-4.

Shyamalamma, S., S. B. C. Chandra, M. Hegde, and P. Naryanswamy. 2008. Evaluation of genetic diversity in jackfruit (Artocarpus heterophyllus Lam.) based on amplified fragment length polymorphism markers. Genetics and Molecular Research 7:645-656.

Srivastav, M., A. K. Dubey and R. R. Sharma.2005. Effect of rootstocks on leaf nutrients, tree growth, yield and fruit quality of Mosambi sweet orange (citrus sinensis) under Delhi condition. Indian Journal of Agricultural Sciences. 75(6):333-335.

Sturrock, D. 1959. Fruits of Southern Florida. South Eastern Printing Co. Stuart. Fla. P. 114.

Ullah, M. A. and M. A. Haque. 2008. Studies on fruiting, bearing habit and fruit growth of jackfruit germplasm. Bangladesh J. of Agril. Res. 33:391-397.

Westwood, M.N., Reimer, F.C. and Quockenbush, V.L. 1963. Long varieties grown on rootstock of five pyrus spp. Proceedings, American Social Horticulure Science., 82:103-108. 
